\title{
Proportional-Integral-Derivative Controllers Tuning for Unstable and Integral Processes Using Genetic Algorithms
}

\author{
Marco Antonio Paz-Ramos ${ }^{1,2}$, Jose Torres-Jimenez ${ }^{3}$, and \\ Enrique Quintero-Marmol-Marquez ${ }^{2}$ \\ 1 Instituto Tecnologico y de Estudios Superiores de Monterrey \\ Campus Ciudad de Mexico Calle del Puente 222 Col. Ejidos de Huipulco \\ Tlalpan, 14380, Mexico, D.F., Mexico marco.paz@itesm.mx \\ 2 Centro Nacional de Investigación y Desarrollo Tecnológico (CENIDET) \\ Interior Internado Palmira S/N Cuernavaca, Morelos, Mexico \\ $\{$ pazramos, eqm\}@cenidet.edu.mx \\ 3 Instituto Tecnologico y de Estudios Superiores de Monterrey Campus Cuernavaca, \\ Computer Science Department. \\ Av. Paseo de la Reforma 182-A. Lomas de Cuernavaca 62589 Temixco Morelos, \\ Mexico \\ jtj@itesm.mx \\ Tel. +52-777-3297169, Fax +52-777-3297168
}

\begin{abstract}
During the last years the use of intelligent strategies for tuning Proportional-Integral-Derivative (PID) controllers has been growing. The evolutionary strategies have won an important place thanks to their flexibility. In this paper, the automatic tuning of systems with scarce initial information and integrative and unstable dynamics, through a genetic approach is presented. The advantages of the proposed approach were highlighted through the comparison with the Ziegler-Nichols modified closed loop method, and the Visioli genetic approach. The proposed methodology goal is to expand the intelligent tuning application to a wider range of processes (covering systems with oscillatory or unstable modes).
\end{abstract}

Keywords: PID Controllers, Genetic Algorithms.

The first attempts to automate the tuning of Proportional-Integral-Derivative (PID) controllers were based on the time response of a process, but this approach had the drawback of requiring a lot of user interaction. A very important advance was made when it was decided to use the frequency response of a process instead of its time response, in this way a bigger degree of automation was attained. The first industrial PID controller based on processes' frequency response, was the Novatune [1, which initialized a PID controller automatically.

Applications based on PID controllers using frequency response were very successful [1] 2] 3], however the range of their applicability is restricted to asymptotically stable systems, due to the use of the relay feedback. By using 
evolutionary meta heuristics, like Genetic Algorithms (GA), it is possible to expand the application range of PID controllers to systems with oscillatory or unstable modes. The application of GA to control applications has been increasing steadily and recently GA were applied: a) to tune Multiple-Input-MultipleOutput (MIMO) systems [4] [5]; and b) to improve significantly the performance of plants [6].

In this paper, a GA was used to automate the tuning of systems with scarce initial information and integrative and unstable dynamics, in order to get insight of the advantages of using a GA, the results were compared against the ZieglerNichols modified closed loop method. The rest of the paper is organized in four more sections: a) in section two the Ziegler-Nichols closed loop control method is presented; b) in section 3 the Visioli genetic method for integral and unstable processes was highlighted; c) in section 4 the Direct Genetic PID proposed method, that is able to complement the Ziegler-Nichols and Visioli methods is presented; and d) finally in section 5 main conclusions will be presented.

\section{Ziegler-Nichols Closed Loop Method}

With the microprocessor arrival, the industrial field controllers' horizons were expanded, thanks to the capacity to carry out elaborated algorithms. This situation encouraged some controller makers to develop auto tuning PID controllers. One example of this controllers is the Foxboro EXACT [7, whose operation was based on the transient response analysis. An alternative was the use of Ziegler and Nichols closed-loop method [7]. The original method consists of varying the proportional gain until a sustained oscillation is reached. Maintaining the system in this state, the amplitude and frequency of the oscillations are determined by measuring the system output. A PID controller has the standard form depicted in equation (1), where $u(t)$ defines the controller output (also called process output), $e(t)$ is the system error (difference among the desired set point for the variable under control, and the actual process variable value), $K_{p}$ indicates the proportional gain, $T_{d}$ is the derivative time constant, and $T_{i}$ is the integral time constant.

$$
u(t)=K_{p}\left(e(t)+\frac{1}{T_{i}} \int_{0}^{t} e(t) d t+T_{d} \frac{d e(t)}{d t}\right)
$$

The PID controller parameters for equation (11) are calculated and fixed according to the table 1 where $K_{u}$ is the ultimate gain, and $T_{u}$ is the ultimate period. The Ziegler and Nichols method for tuning an ISA PID controller, is a thumb rule based on the Nyquist curve. According to the Nyquist theorem a process is stable if the Nyquist curve does not encircle the point $(-1,0)$.

\subsection{Using the Relay Feedback System}

Hägglund and Aström [1] [9] introduced an alternative to the increment of the proportional gain to achieve the oscillations inside a bigger margin of security 
Table 1. Regulator parameters obtained by the Ziegler-Nichols closed-loop method

\begin{tabular}{|l|l|l|l|}
\hline Controller & $\mathbf{K}_{\mathbf{p}}$ & $\mathbf{T}_{\mathbf{i}}$ & $\mathbf{T}_{\mathbf{d}}$ \\
\hline $\mathrm{P}$ & $0.5 K_{u}$ & & \\
$\mathrm{PI}$ & $0.4 K_{u}$ & $0.8 T_{u}$ & \\
$\mathrm{PID}$ & $0.6 K_{u}$ & $0.5 T_{u}$ & $0.12 T_{u}$ \\
\hline
\end{tabular}

that the original free oscillations system. A non linear circuit was introduced. The non linear function has a relay characteristic. To obtain the value of the proportional gain according to the table 1 is necessary to calculate the equation (2) where $d$ is the output relay amplitude and a is the oscillation amplitude, while $T_{u}$ (necessary to obtain $T_{i}$ and $T_{d}$ ) is measured directly of the oscillations.

$$
K_{u}=\frac{4 d}{a \pi}
$$

\subsection{Stability of Limit Cycle}

The relay feedback method is successfully used in the industry [1], however to measure $K_{u}$ and $T_{u}$ is fundamental that the limit cycle were stable and symmetric. Asström [8] developed an analysis consisting in obtaining the Jacobian of the Poincaré map $(W)$ which is related to the relay oscillations; the limit cycle is locally stable if and only if $W$ has all its eigenvalues inside the unit disk. The phase portrait is a practical way to know if a process oscillation is stable or not, when the relay feedback is applied. Suppose the plant described in equation (3). The phase portrait of equation (3) obtained during the relay experiment is shown in the figure 1(a). We can observe in the phase portrait that exists a stable limit cycle.

$$
G(s)_{1}=\frac{1}{(s+1)^{3}}
$$

Now, if we take the phase portrait of the process represented in equation (44), the limit cycle is unstable, and it can be observed in figure प(b). It is possible to obtain similar results for processes described in equations (5) and (6), given that, their limit cycles are stable but no asymptotically stable [8] (also these processes are unstable when the feedback relay is used).

$$
\begin{gathered}
G(s)_{2}=\frac{1}{s^{3}+s^{2}+s+1} \\
G(s)_{3}=\frac{1}{s^{2}} \\
G(s)_{4}=\frac{1}{s^{2}+1}
\end{gathered}
$$




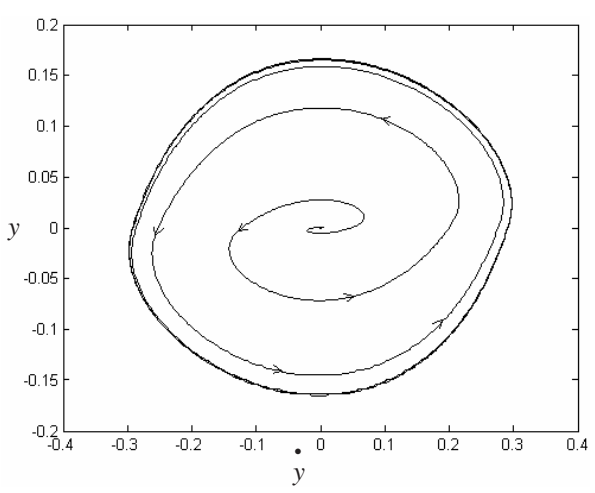

(a)

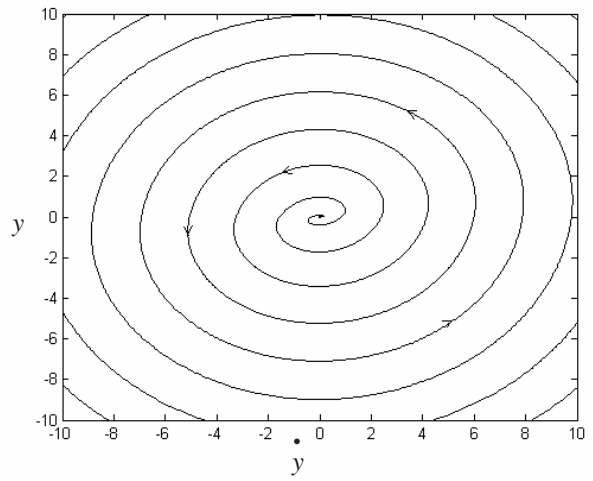

(b)

Fig. 1. (a) Phase portrait during the relay experiment for equation (3), (b) Phase portrait during the relay experiment for equation (4).

Generally the integrative and unstable processes do not allow reaching a controlled oscillation. If do not exist a stable oscillation it is not possible to apply the Ziegler and Nichols methods, one of the alternatives to tuning PID controllers for integral and unstable processes is the use of GA. The Visioli method [12] presented in next section uses internally a GA.

\section{Visioli Genetic Method for Integral and Unstable Processes}

In this section, a brief introduction to GA concepts will be presented first, and then the Visioli method (based on GA) is detailed. The original idea of GA was to achieve that the computing processes could have an evolutionary profile. In the last years there has been an important increase of genetic algorithms applications to the automatic control field. A genetic algorithm emulates an evolutionary process [1], thanks to a generation of virtual individuals population. The virtual population has a reproduction and death process, which allow space for a new generation. According to the schemata theorem [11] if reproduction possibilities for the better adapted individual are increased, the future generations will be more capable on the average than their predecessors. If we take the population individuals as potential solutions of a problem, and we build the algorithm so that the most capable individuals are the nearest to the solution, then we can hope after a certain number of generations, the solution to our problem.

Visioli presents in 12 a methodology for tuning PID controllers for integral and unstable processes. The method consists on a set of rules for integral and unstable processes, which are similar to the Ziegler and Nichols rules. These rules were obtained using a Genetic Algorithm optimization approach. The method contemplates the structure described in equation (7) for integrative processes 
( $K$ is the transfer function gain, and $L$ is the system delay), and the structure described in equation (8) for unstable processes ( $T$ is a time response rate).

$$
\begin{gathered}
G(s)=\frac{K}{s} e^{-L s} \\
G(s)=\frac{K}{T s-1} e^{-L s}
\end{gathered}
$$

In a Visioli paper 12 tables for tuning rules for optimal set-point response for unstable processes can be found. The rules are optimized to minimize the integral of the quadratic error (ISE).

Suppose an unstable plant whose transfer function is given by the equation (9). In accordance with Visioli rules [12], the parameter values will be: $K_{p}=5.8$, $T_{i}=1.88$, and $T_{d}=0.11$. In the figure 4 (a) the response of the process described in equation (9) when is controlled by the equation (11) is shown.

$$
G(s)_{5}=\frac{1}{s-1} e^{-0.2 s}
$$

The main virtue of the Visioli method is its simplicity of use; however it has three important disadvantages: a)an identified model of the process is needed; b) the identified model has to be of first order approach; and c) If $L=0$ ( $L$ is the system delay), the method can not be used. In the next section we propose a methodology for tuning a PID control for integral and unstable process which is independent to the transfer function order. The method is a direct optimization of the controller parameters across a genetic algorithm, as it will be shown this method is superior to the Visioli approach.

\section{Direct Genetic PID}

The use of genetic algorithms for tuning PID controllers has been growing, because is very easy to connect the structure of the controller with the optimization strategy. The genetic algorithm task is to optimize the controller parameters, by means of an evolutionary process. Suppose a chromosome coded as is indicated in equation (10), where $C_{k}$ is the gain chromosome. $C_{k}$ represents a simple individual whose genes are the PID controller parameters $\left(C_{k}=K_{p} T_{i} T_{d}\right.$, $K_{p}=p_{1}, p_{2}, \ldots, p_{n}, T_{i}=i_{1}, i_{2}, \ldots, i_{n}$, and $\left.T_{d}=d_{1}, d_{2}, \ldots, d_{n}\right)$. Every chromosome is tested and evaluated. The individuals with fitness higher than the average shall have more reproduction opportunities. The gains that allow a better control performance give more chance to inherit the chromosome profile. To optimize the search, the decoding indicated in equation (11) is carried out. In equation (11), $A_{i}$ is a gene of the chromosome $C_{k}$, which is weighted by a decoder weight $i$, fixed in a heuristic way (see 4]), and $K_{i}$ is a gain used by the equation (11). In the figure 2 is shown the optimization process, and it can be observed an on line identification process which use recursive least squares (RLS). The intern 


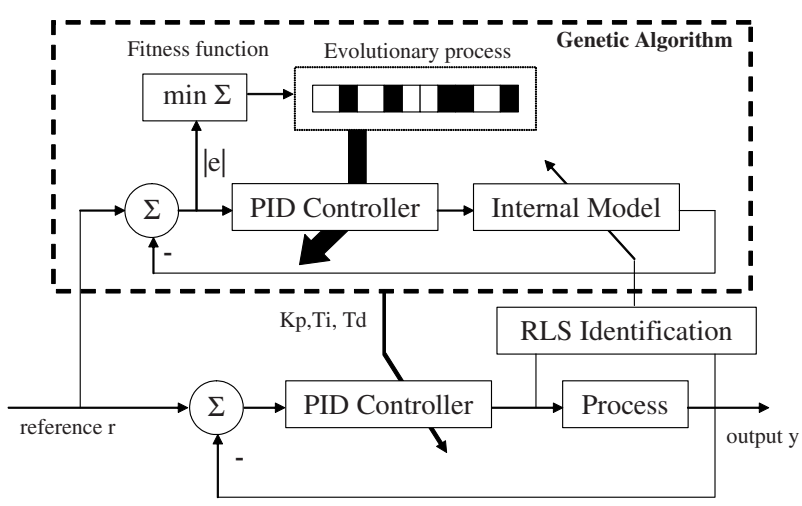

Fig. 2. Optimization structure

model is used in the fitness function described in equation (12), where $r_{i}$ is the reference point, $\widehat{y}$ is the estimated process and $n$ is a value that allows the output process establishment, when it is stable otherwise $n$ will have a default value. In the equation (12), the minimization criterion is the integral of absolute error (IAE).

$$
\begin{gathered}
C_{k}=p_{1}, p_{2}, \ldots, p_{n}, i_{1}, i_{2}, \ldots, i_{n}, d_{1}, d_{2}, \ldots, d_{n} \\
K_{i}=\Delta_{i} A_{i} \\
F F=\sum_{i=0}^{n}\left|r_{i}-\widehat{y}\right|
\end{gathered}
$$

The higher qualified individuals are those that minimize the equation (12). The controller parameters are modified using a commutation only when an important parameter variation happens.

The genetic algorithm for the optimization has a population of 100 members. In every reproduction process a tournament selection is performed involving $10 \%$ of population. The maximum allele value is $9.0072 \times 10^{15}$. For maximum focus in the search, decoding considers the value dividing the allele between ten times its maximum value. A mutation process which involves $10 \%$ of the population is applied.

Previously, it was stated that the process described in equation (4) does not have a stable limit cycle; therefore it is not possible to use the Ziegler and Nichols methods. Applying the Direct Genetic PID, it is possible to control a marginally stable process. In the figure 3(a), it is shown the plant controlled output and the evolutionary process evolution. The resultants gains for the process defined in equation (44) are $K_{p}=0.156, T_{i}=0.312$ and $T_{d}=9.96$. 
The genetic optimization to control the double integrative process described in equation (5) is presented in figure 3(b). The resultants gains for the process described in equation (515) are $K_{p}=4.251, T_{i}=99.16$ and $T_{d}=0.781$. Also is shown the control for the unstable delayed process described by equation (9) in figure 4(b). The resultants gains for the process described in equation (9) are $K_{p}=2.910, T_{i}=1.367$ and $T_{d}=0.135$.
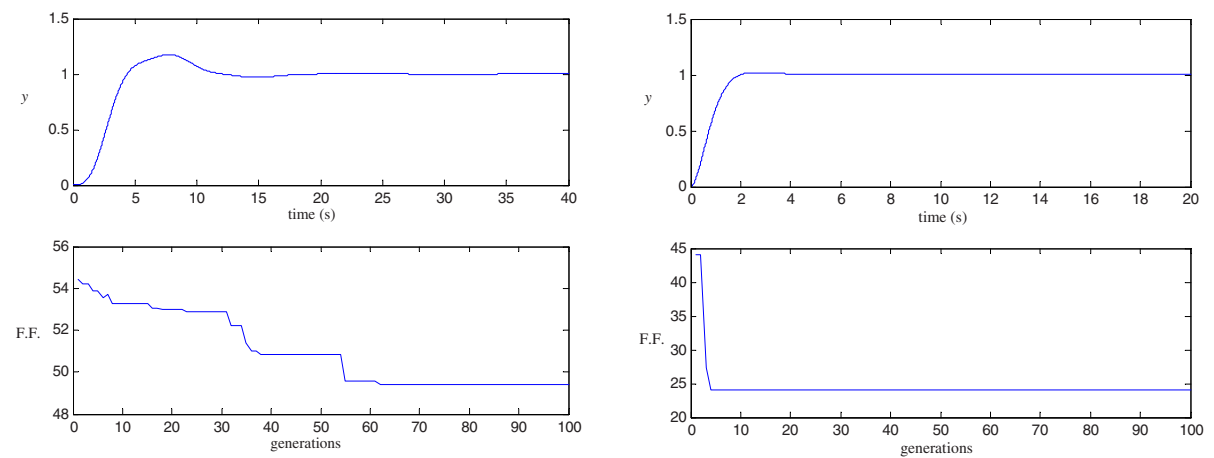

(a)

(b)

Fig. 3. (a) Genetic PID control of the process described in equation (4), (b) Genetic PID control of the process described in equation (5).

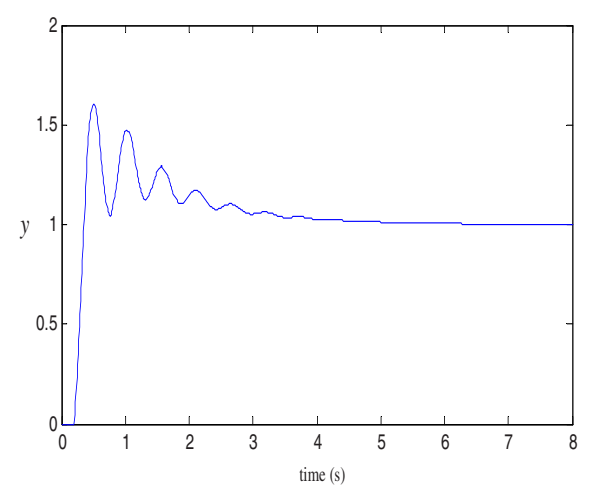

(a)
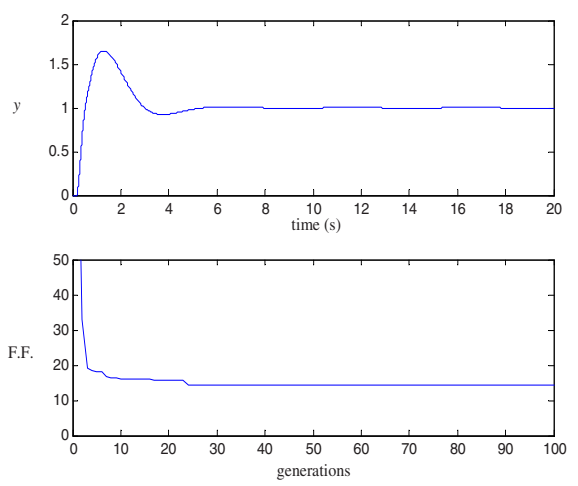

(b)

Fig. 4. (a) PID Control of the process described in equation (9) using Visioli method, (b) PID control of the process described in equation (9) using the proposed method. 


\section{Conclusions}

In this paper a new Genetic PID approach was presented to make control of systems with oscillatory or unstable modes. The main advantages of the presented method relate to the possibility of make PID tuning when other methods have failed (the Visioli method is not able to handle the case when $L=0$ meanwhile the proposed method is capable to handle this case). Satisfactory results were observed through the exhibited tests in the paper. It is intended that the Genetic PID approach can be connected easily as a backup controller for other adaptive controllers. It is remarkable that the Genetic PID approach proposed does not require to know the process features, because an estimation stage (RLS) can be used.

\section{References}

1. Häglund T., and Åström K. J., Industrial Adaptive Controllers Based on Frequency Response Techniques, Automatica, Vol. 27, No.4, (1991) 594-609.

2. Hägglund T, and. Åström K.J., Supervision of Adaptive Control Algorithms, Automatica. Vol. 36, (2000) 1171-1180.

3. Isermann R., and Lachmann K.-H., Parameter-adaptive Control with Configuration Aids and Supervision Functions, Automatica, Vol. 21, No. 6, (1984) 625-638.

4. Vlachos C., Williams D., and Gomm J. B., Genetic Approach to Decentralized PID Controller Tuning for Multivariable Processes, IEE Proc. Control theory Appl. Vol 146, No. 1, (1999) 58-64.

5. Paz R. M.A., García B. C. D., and Torres J. J., Fuzzy-Genetic Controller for a Coupled Drives System, IEEE International Symposium on Industrial Electronics, Puebla, Mexico (2000) 741-746.

6. Krohling R. A., and Rey UJ. P., Design of Optimal Disturbance Rejection PID Controllers Using Genetic Algorithms, IEEE Transactions on evolutionary computation. Vol 5, No. 1, (2001) 78-82.

7. Åström K. J., and Hägglund T., PID Controllers: Theory, Design, and Tuning ISA organization 1995, ISBN: 1556175167

8. Åström K. J. et al (ed.), Adaptive Control, Filtering, and Signal Processing, Springer-Verlag 1995,ISBN: 0387979883

9. Hägglund T., and Åström K. J., Method and Apparatus in Tuning a PID-Regulator, U.S. Patent. Number 4549123, 1995

10. Holmberg V. Relay, Feedback of Simple Systems, Ph.D. Thesis, August 1991, Department of Automatic Control Lund Intitute of Technology

11. Goldberg D., Genetic Algorithms in Search, Optimization and Machine Learning, Addison Wesley 1989,ISBN: 0201157675

12. Visioli A., Optimal Tuning of PID Controllers for Integral and Unstable Processes, IEEE Proc. -Control Theory Appl., Vol. 148, No. 2. (2001) 180-184. 